\title{
ESTRATÉGIAS DE MONITORAMENTO NO ENFRENTAMENTO DA COVID-19 EM SOBRAL-CEARÁ
}

\author{
MONITORING STRATEGIES TO COPE WITH COVID-19 IN SOBRAL-CEARÁ \\ ESTRATEGIAS DE MONITOREO EN EL COMBATE A LA COVID-19 EN SOBRAL-CEARÁ
}

\author{
Josiane Alves Dorneles 1 \\ Rogeriany Lopes Farias ${ }^{2}$ \\ Larissa Cavalcante Fonteles Araújo 3 \\ Paloma de Vasconcelos Rodrigues ${ }^{4}$ \\ Isabelle Frota Ribeiro Queiroz ${ }^{5}$ \\ Germana Maria da Silveira 6
}

Palavras-chave:

Monitoramento Epidemiológico; Infecções por Coronavírus; Consulta Remota

Keywords: Epidemiological Monitoring; Coronavirus Infections; Remote Consultation.

Palabras clave: Monitoreo Epidemiológico; Infecciones por Coronavirus; Consulta Remota.

Submetido: $14 / 11 / 20$

Aprovado: $17 / 12 / 20$

Autor(a) para Correspondência: Rogeriany Lopes Farias Rua Maria da Conceição P. de Azevedo, 1206, Renato Parente - Sobral - CE CEP:62.033-170

E-mail: rogerianylopes@gmail.com

\section{RESUMO}

0 presente trabalho se trata de um estudo descritivo, do tipo relato de experiência, com abordagem qualitativa, que objetiva descrever as principais estratégias de monitoramento e vigilância adotadas pela Secretaria de Saúde do Municipio de Sobral-Ceará no enfrentamento da pandemia de Covid-19, realizado no período de março a setembro de 2020. Serão abordadas, neste estudo, as ações estratégicas implementadas com vistas ao efetivo monitoramento dos casos suspeitos e confirmados e oferecer retaguarda à gestão municipal para a tomada de decisão, a saber: implantação de uma Central de Monitoramento da Covid-19, monitoramento descentralizado dos casos pela Atenção Primária à Saúde e criação do teleatendimento por meio do Plantão Epidemiológico 24 horas. Essas estratégias foram fundamentais para subsidiar a gestão municipal no planejamento de ações e fluxos intersetoriais necessários para o enfrentamento da pandemia. Dessa maneira, revestiram-se de relevância ao assegurarem adequado acompanhamento das condições de saúde da população, a prevenção de agravos e controle da transmissibilidade da doença.

1. Médica. Mestre em Saúde da Família (UVA/RENASF). Especialista em Ginecologia e Obstetrícia (Hospital Fêmina do Grupo Hospitalar Conceição - GHC). Assistente Técnica da Atenção Primária à Saúde de Sobral(2017-2020). Secretaria da Saúde de Sobral. Prefeitura de Sobral. E-mail: josianealvesdorneles2009@hotmail.com. 0RCID: http://orcid.org/0000-0002-5856-2941

2. Assistente Social. Mestranda em Saúde da Família (UVA/RENASF). Gerente da Atenção Primária à Saúde de Sobral. Secretaria da Saúde de Sobral. Prefeitura de Sobral. E-mail: rogerianylopes@gmail.com. ORCID: https:// orcid.org/0000-0003-1040-727X

3. Enfermeira. Especialista em Saúde da Família (UNINTA). Especializanda em Gestão e Auditoria em Sistemas de Saúde e em Preceptoria no SUS (Śrio Libanês). Coordenadora da Atenção Primária de Sobral. Secretaria da Saúde de Sobral. Prefeitura de Sobral. E-mail: larissacfontelesaraujo@gmail.com.0RCID: http://orcid.org/0000-0003$\underline{1961-4099}$

4. Enfermeira. Mestranda em Saúde da Família (UVA/RENASF). Docente da Escola de Saúde Pública Visconde de Saboia (ESP-VS).E-mail: palomavasconcelos@hotmail.com.0RCID: http://orcid.org/0000-0003-0066-1485

5. Enfermeira. Mestranda em Saúde da Família (UFC). Gerente do Centro de Saúde da Família Junco. Secretaria da Saúde de Sobral. Prefeitura de Sobral. E-mail: isabellefrota2010@hotmail.com. ORCID: http://orcid.org/00000002-9259-8833

6. Enfermeira. Mestre em Cuidados Clínicos em Enfermagem e Saúde pela Universidade Estadual do Ceará (UECE). E-mail: germanasilveira@hotmail.com.0RCID: http://orcid.org/0000-0003-3471-5525 


\section{ABSTRACT}

The present work is a descriptive experience report type OF study, with a qualitative approach, which aims to describe the main monitoring and surveillance strategies adopted by the Secretaria de Saúde, in the municipality of Sobral-Ceará, in the fight against the Covid-19 pandemics, during the period from March to September 2020. It will discuss strategic actions implemented with the objective of effective monitoring of suspected and confirmed cases and offer a backup to municipal management for decision making, namely: Implementation of a Covid-19 Monitoring Center; Decentralized monitoring of cases by Primary Health Care, and the creation of a call center through the 24-hour Epidemiological Service. These strategies were essential to support municipal management in planning actions and intersectoral flows necessary to face the pandemics. Thus, they were relevant to ensure adequate monitoring of the population's health conditions, the prevention of diseases, and the control of disease transmissibility.

\section{RESUMEN}

El presente trabajo se trata de un estudio descriptivo, del tipo relato de experiencia, con enfoque cualitativo, que pretende describir las principales estrategias de monitoreo y vigilancia adoptadas por la Secretaría de Salud, del municipio de Sobral-Ceará, en el enfrentamiento a la pandemia Covid-19, en el período de marzo hasta septiembre de 2020. Discurrirá acciones estratégicas aplicadas en vistas al efectivo monitoreo de los casos sospechosos y confirmados y ofrecerá retaguardia a la gestión municipal para la tomada de decisión, a saber: Implantación de una Central de Monitoreo de la Covid-19; Monitoreo descentralizado de los casos por la Atención Primaria a la Salud y creación de un centro de atención al cliente por medio de Servicio Epidemiológico 24horas. Esas estratégicas fueron fundamentales para subsidiar la gestión municipal en el planeamiento de acciones y flujos intersectoriales necesarios para el combate a la pandemia. De esta forma, se revistieron de relevancia al garantizar adecuado acompañamiento de las condiciones de salud de la población, la prevención de agravios y el control de la transmisibilidad de la enfermedad.

\section{INTRODUÇÃO}

0 enfrentamento da pandemia causada pelo vírus SARS-CoV-2 (do inglês: Servere Acute Respiratory Syndrome Coronavirus 2), responsável pela Covid-19, representa um desafio para a ciência e para a sociedade, exigindo respostas imediatas e adaptação do sistema de saúde ${ }^{1}$. Na ausência de vacina e tratamentos específicos, torna-se fundamental a adoção de estratégias de isolamento, distanciamento social, monitoramento dos casos e de compartilhamento de informações a fim de reduzir a transmissão e conter esse agravo ${ }^{2}$.

0 cenário epidemiológico mundial provocado pela pandemia exigiu dos equipamentos de saúde uma reorganização na forma de cuidado e atenção. A criação e articulação de ações foram necessárias para garantir a manutenção dos serviços, a proteção dos trabalhadores e a contenção do avanço de transmissão viral. Com isso, houve uma necessidade imediata de redefinição de objetivos, ações prioritárias e resolutivas capazes de enfrentar com celeridade e eficácia a condição epidemiológica atual.

Dentre essas ações, é possível destacar a criação de estratégias de monitoramento e rastreamento de contatos, as quais são consideradas importantes medidas de saúde pública que visam à diminuição da propagação de doenças infectocontagiosas a partir da identificação de novas infecções resultantes da exposição a um caso conhecido ${ }^{3}$.

Nesse sentido, o município de Sobral, no estado do Ceará, percebeu a necessidade de desenvolver ações estratégicas de monitoramento da Covid-19 no intuito de acompanhar o crescimento dos índices de contágio e os locais de maior concentração de pessoas infectadas, de avaliar a evolução dos sintomas dos pacientes para prevenir agravos e subsidiar o atendimento na rede de saúde, além de ampliar o acesso da população à informação e ao acompanhamento por profissionais de saúde, sem sair de casa. Tais ações foram fundamentais para a tomada de decisões da gestão municipal, bem como para a prevenção, tratamento e controle da doença.

Diante do exposto, o presente trabalho busca descrever as principais estratégias de monitoramento e vigilância adotadas pelo município de Sobral-Ceará no enfrentamento da pandemia de Covid-19. 


\section{METODOLOGIA}

0 presente trabalho se trata de um estudo descritivo, do tipo relato de experiencia, com abordagem qualitativa, que objetiva apresentar as estratégias adotadas pelo município de Sobral no que se refere ao monitoramento de casos de Covid-19, nos meses de março a setembro de 2020, tais como a criação da Central de Monitoramento Covid-19, o Monitoramento Descentralizado na Atenção Primária à Saúde (APS) e a criação do Plantão Epidemiológico 24 horas.

0 município de Sobral, local de referência para este estudo, iniciou as estratégias de enfrentamento da Covid-19 de modo precoce. A cidade registrou 0 seu primeiro caso da doença no início de março, mas antecipou parte de suas ações, pautadas nas experiências de outros países, ainda em fevereiro, quando foi notificado o primeiro caso suspeito, que futuramente fora descartado.

Desse modo, dedicou-se ao planejamento de ações e fluxos intersetoriais fundamentais para o enfrentamento da pandemia, com o objetivo de apoiar as ações de atenção à saúde, monitorar a transmissibilidade do vírus e subsidiar estratégias de controle e prevenção da doença, fortalecendo a capacidade do sistema de saúde do município para responder ao novo coronavírus.

\section{RESULTADOS E DISCUSSÃO}

A Secretaria Municipal de Saúde (SMS) de Sobral, diante do cenário da pandemia de Covid-19, reuniu uma série de ações e estratégias articuladas entre os diversos setores das políticas públicas no intuito de reduzir a transmissão do vírus e prestar assistência à população, especialmente àquela que mais precisava. Dentre essas, serão destacadas a criação da Central de Monitoramento Covid-19, a estratégia de Monitoramento Descentralizado na Atenção Primária à Saúde e a criação do teleatendimento Plantão Epidemiológico 24 horas.

\section{Central de Monitoramento Covid-19}

Uma das medidas desenvolvidas no âmbito do planejamento e coordenação das ações pela Secretaria Municipal de Saúde foi a criação, no mês de março de 2020, de uma Central de Monitoramento Covid-19. Tal medida foi pensada como uma estratégia centralizada de monitoramento e vigilância de casos suspeitos ou confirmados para a Covid-19.

A Central de Monitoramento, em sua formação, contou com a participação de profissionais gestores vinculados à SMS, profissionais do Núcleo Ampliado de Saúde da Família e Atenção Básica (Nasf-AB) e profissionais dos dois programas de Residências Multiprofissionais em Saúde da Família (RMSF) e em Saúde Mental (RMSM), da Escola de Saúde Pública Visconde de Saboia (ESP-VS), contabilizando 31 profissionais. A Central funcionava de 07:00h às 19:00h, durante os sete dias da semana, com organização de trabalho em escalas entre os profissionais.

0 trabalho dos profissionais da Central de Monitoramento era pautado na intersetorialidade como linha prioritária de ação. A comunicação entre os diversos segmentos da saúde municipal era constante e efetiva.

Com base nas conjecturas teóricas e metodológicas da promoção da saúde, o trabalho interprofissional aparece como instrumento relevante para a atuação do conceito ampliado de ações de saúde, configurandose como ação-chave no trabalho em equipe ${ }^{4}$.

Os serviços realizados pela equipe de monitoramento compreendiam a notificação dos casos suspeitos ou confirmados para a Covid-19 no sistema de informação do Ministério da Saúde (MS), e-SUS Vigilância Epidemiológica (e-SUS VE), o preenchimento de planilhas de informação e controle local criadas pelo Comitê de Crise Covid-19 de Sobral, que continham dados pessoais e epidemiológicos de todos os casos notificados, as quais serviam de instrumento para a confecção do boletim diário da situação epidemiológica do município, e a interlocução com a rede de serviços da Atenção Básica, as unidades hospitalares, os laboratórios, farmácias e empresas.

A comunicação com essas redes acontecia constantemente e tinha o intuito de notificar casos identificados com quadro clínico suspeito ou confirmado para a Covid-19; na oportunidade,

\section{"O trabalho dos} profissionais da Central de Monitoramento era pautado na intersetorialidade como linha prioritária de ação" 
eram alinhados os fluxos organizacionais e compartilhadas as situações específicas de usuários com a Atenção Primária, que realizava as avaliações iniciais com estratificação dos casos, bem como a realização do manejo clínico para os casos leves; e também a coordenação do cuidado para os casos que necessitavam de atenção especializada. Esse conjunto de ações contribuía para a garantia da detecção, avaliação e tempo de resposta adequados ao cuidado e evolução da Covid-19.

0 agendamento dos testes diagnósticos para a Covid-19 ficou também sob responsabilidade da equipe da Central de Monitoramento. Desde o início da transmissão comunitária no município, lançou-se especial atenção para a capacidade de testagem para a detecção dos casos, de modo a conter a transmissão da Covid-19.

Diante do contexto da pandemia, por se tratar de um vírus novo e em processo de descobertas, várias foram as dificuldades enfrentadas durante o percurso da Central de Monitoramento. Com isso, pode-se destacar os principais desafios encontrados, como as constantes alterações dos protocolos de vigilância e atenção à Covid-19, que incluíam sempre um novo conjunto de recomendações no campo da prevenção e Vigilância em Saúde, exigindo um processo de aprendizagem rápido e diversas reorganizações de fluxos e dos processos de trabalho do grupo. Além disso, a rotina intensa de trabalho, que crescia de acordo com o aumento das taxas de contágio, tornou-se também um dos grandes desafios vivenciados, exigindo de todos os profissionais uma maior atenção, esforço e dedicação.

Com a centralização e as demais ações determinadas pela Central de Monitoramento, como o rastreio precoce com ações realizadas nos territórios, testagem e acompanhamento dos casos confirmados e suspeitos, Sobral intensificou 0 monitoramento, rastreamento e o bloqueio dos casos de Covid-19. De acordo com os dados mundiais sobre o tema, o rastreamento e monitoramento de pessoas infectadas pela Covid-19 e de seus contatos provou ser uma das formas mais eficazes de reduzir a disseminação do vírus. Segundo a Organização Pan-Americana da Saúde (OPAS), países que estão em fase mais adiantada da doença utilizaram essa estratégia com sucesso ${ }^{5}$.

Dessa maneira, foi possivel observar que a Central de Monitoramento se mostrou fundamental para o enfrentamento da Covid-19 no município de Sobral, pois colaborou para o acompanhamento

\section{“0 agendamento dos testes diagnósticos para a Covid-19 ficou também sob responsabilidade da equipe da Central de Monitoramento"}

integral das famílias e dos pacientes acometidos ou suspeitos de contaminação pelo novo coronavírus na Atenção Primária e subsidiou diretamente a tomada de decisões das autoridades sanitárias no município.

\section{Monitoramento descentralizado na Atenção Primária à Saúde de Sobral, Ceará}

Considerando que a Atenção Primária à Saúde também exerce papel central no enfrentamento da Covid-19, uma vez que está ancorada nos princípios de proteção da saúde, na prevenção e controle de doenças, sendo capaz de colaborar estrategicamente com a redução do risco de transmissão da doença a partir do diagnóstico precoce, acompanhamento e monitoramento individual e familiar ${ }^{6}$, foi necessário planejar a organização dos Centros de Saúde da Família (CSF) para garantir o acesso e o acolhimento de todos os pacientes sintomáticos respiratórios, no período da pandemia de Covid-19, desenvolvendo atividades curativas e de acompanhamento clínico baseadas nas evidências científicas disponíveis no momento; assim como o monitoramento descentralizado dos pacientes acometidos, destacando-se a descentralização, como a realização do monitoramento por cada equipe da Estratégia Saúde da Família (ESF) de sua população adstrita.

Um dos elementos essenciais do acompanhamento clínico proposto para os sintomáticos respiratórios, no período da pandemia, foi o monitoramento dos casos identificados pelas equipes da ESF de Sobral. Tal iniciativa está de acordo com as diretrizes da Política Nacional de Atenção Básica (PNAB) e os princípios da Atenção Primária, levando em consideração o conhecimento das condições de vida e da saúde de cada comunidade.

Esse monitoramento foi fundamentado nos princípios da APS, do primeiro contato, longitudinalidade, integralidade, coordenação do cuidado, abordagem familiar e enfoque comunitário ${ }^{7}$, associados à fundamental normatização do fluxo dos 
usuários na rede de atenção e o estabelecimento de rotinas internas em cada unidade de saúde componente da rede de atenção.

Os casos eram monitorados pelas equipes da ESF, sendo classificados como casos leves, conforme o Ministério da Saúde $(M S)^{8}$, aqueles em que o cidadão apresentava síndrome gripal com sintomas leves, na ausência de comorbidades que contraindicassem o isolamento domiciliar e também não apresentassem sinais de gravidade, como dispneia, saturação de $0_{2}<95 \%$, taquipneia, hipotensão, piora nas condições clínicas basais e alteração do estado mental.

Após a classificação, aos casos leves era orientado o manejo clínico com prescrição de medicações sintomáticas, orientações de isolamento domiciliar, de uso de máscaras e monitoramento diário, por telefone, até o paciente completar 14 dias do início dos sintomas ou apresentar agravamento do quadro clínico ou alguma complicação clínica. No decorrer dos 14 dias de monitoramento, se algum familiar desenvolvesse sintoma, eram agendados atendimentos para evitar os riscos da aglomeração nos CSF. Também eram fornecidos atestados para o paciente e contatos domiciliares por 14 dias, a contar do início dos sintomas.

Os integrantes das equipes da Estratégia de Saúde responsáveis pelo monitoramento eram os profissionais médicos, enfermeiros, dentistas e agentes comunitários de saúde (ACS) que, no momento, encontravamse afastados das atividades laborais por pertencerem aos grupos de risco para a Covid-19, ou por estarem impossibilitados de realizar suas atividades rotineiras, por conta das características de contágio dessa nova doença.

0 monitoramento dos casos leves era realizado preferencialmente por telefone. Desse modo, quanto àqueles pacientes que fazem parte do grupo de risco para a Covid-19 ou àqueles sem acesso a telefone, 0 monitoramento era realizado por meio de visita domiciliar. Com o agravamento da pandemia no município de Sobral e a impossibilidade de ofertar testagem para todos os sintomáticos, foram instituídas duas modalidades de monitoramento dos casos leves, com o objetivo de detectar em tempo oportuno as complicações e reduzir a morbidade e a mortalidade nesse grupo de pacientes. Assim, os casos leves passaram a ser monitorados por telefone ou visita domiciliar, conforme pode ser observado no Quadro 1.

Quadro 1 - Frequência de monitoramento dos casos leves de Covid-19. Sobral, Ceará, 2020.

\begin{tabular}{|l|}
\hline Covid-19 positivo - visita domiciliar diária por 14 dias \\
\hline Casos suspeitos (Síndrome Gripal) até completar 14 dias \\
\hline Com comorbidade - visita domiciliar diariamente 80 anos - visita domiciliar diariamente \\
\hline Profissionais de saúde - monitoramento telefônico diariamente \\
\hline Profissionais de segurança - monitoramento telefônico diariamente \\
\hline Gestantes de alto risco e puérperas - monitoramento telefônico diariamente \\
\hline Idosos (60 a 79 anos) - monitoramento telefônico diariamente \\
\hline Criança menores de 5 anos - monitoramento telefônico diariamente \\
\hline 0s demais (que não são notificados) - monitoramento telefônico de $2 / 2$ dias \\
\hline
\end{tabular}

Fonte: Elaborado pelas autoras.

Para a realização das visitas domiciliares pelos agentes comunitários de saúde, foram elaborados um instrutivo e um documento para preenchimento diário do acompanhamento prestado a cada paciente acometido. Essas ferramentas continham orientações para a realização segura das visitas domiciliares de monitoramento dos casos leves de Covid-19 e dos sintomáticos respiratórios (Quadro 2). 
Quadro 2 - Recomendações para os Agentes Comunitários de Saúde na pandemia de Covid-19. Sobral, Ceará, 2020.

- Não realizar atividades dentro do domicílio. A visita estará limitada apenas à área peridomiciliar

(frente, lados e fundo do quintal ou térreo).

- Manter distanciamento do paciente de no mínimo um metro; não havendo possibilidade de

distanciamento, utilizar máscara cirúrgica e protetor facial.

- Visualizar o paciente monitorado.

- Perguntar sobre a presença de sinais e sintomas do caso confirmado e de seus familiares.

- Registrar as informações sobre o caso confirmado no formulário específico.

- Se algum familiar do caso confirmado apresentar sinais e sintomas respiratórios, comunicar verbalmente

à equipe de saúde do território e anotar no campo de observação do formulário específico.

- Orientar o paciente monitorado e sua família sobre as medidas de isolamento domiciliar.

- Enviar, por foto no WhatsApp "Grupo de Monitoramento dos Casos confirmados Covid-19", o formulário

específico preenchido imediatamente.

- Higienizar as mãos com álcool em gel $70 \%$.

Fonte: Adaptado das recomendações para adequação das ações dos Agentes Comunitários de Saúde frente à atual situação epidemiológica referente à Covid-19, Ministério da Saúde9.

0 documento para ser preenchimento diariamente na visita domiciliar de acompanhamento dos casos leves e sintomáticos respiratórios de Covid-19 (Figura 1) foi estruturado com um espaço, na parte da frente, destinado à identificação do paciente e do Centro de Saúde da Família, do período da doença e dos sintomas apresentados; no verso, era descrito um conjunto de orientações que o ACS deveria passar aos pacientes e seus familiares, com o intuito de educar as famílias para a prevenção da disseminação da Covid-19 no território.

Figura 1 - Instrumento de Monitoramento dos casos na Atenção Primária à Saúde. Sobral, Ceará, 2020.

- Frente -

\begin{tabular}{|c|c|}
\hline \multicolumn{2}{|l|}{ Nome do paciente: } \\
\hline \multicolumn{2}{|l|}{ CSF: } \\
\hline \multicolumn{2}{|c|}{ PERÍODO DE DOENÇA (Anotar a data da visita realizada) } \\
\hline \multicolumn{2}{|c|}{ DIA 1 (Data do inicio dos sintomas): } \\
\hline DIA 2 & DIA 3 \\
\hline DIA 4 & DIA 5 \\
\hline DIA 6 & DIA 7 \\
\hline DIA 8 & DIA 9 \\
\hline DIA 10 & DIA 11 \\
\hline DIA 12 & DIA 13 \\
\hline \multicolumn{2}{|c|}{ DIA 14 (Data do encerramento dos sintomas): } \\
\hline \multicolumn{2}{|c|}{$\begin{array}{l}\text { SINAIS E SINTOMAS REFERIDOS PELO PACIENTE } \\
\text { (Assinalar os sintomas referidos) }\end{array}$} \\
\hline \begin{tabular}{l|l} 
Febre $\left(>=37,8^{\circ} \mathrm{C}\right)$ \\
aferida ou referida
\end{tabular} & Tosse \\
\hline $\begin{array}{l}\text { Falta de ar } \\
\text { (Dispneia) }\end{array}$ & $\begin{array}{l}\text { Dor muscular } \\
\text { (Mialgia) }\end{array}$ \\
\hline $\begin{array}{l}\text { Fraqueza } \\
\text { (Fadiga) }\end{array}$ & Espirro \\
\hline Dor de garganta & Diarreia \\
\hline $\begin{array}{l}\text { Assintomático, } \\
\text { sem queixas }\end{array}$ & Familiar com queixas \\
\hline
\end{tabular}

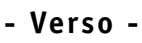

\section{MEDIDAS DE PREVENÇÃO}

(Devem ser orientadas para a familia visitada)

Ainda não existe vacina para prevenção de infecção por COVID-19, portanto, a melhor maneira de prevenir é evitar a exposição ao vírus. Veja algumas maneiras:

- Realizar lavagem frequente das mãos com água e sabão, especialmente após contato direto com pessoas doentes;

- Utilizar lenço descartável para higiene nasal;

- Cobrir nariz e boca quando espirrar ou tossir;

-Evitar tocar mucosas de olhos, nariz e boca;

- Higienizar as mãos após tossir ou espirrar;

- Não compartilhar objetos de uso pessoal, como talheres, pratos, copos ou garrafas;

-Manter os ambientes bem ventilados;

- Permanecer em isolamento domiciliar evitando o contato com pessoas de fora do seu domicílio.

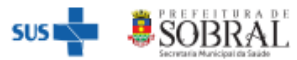

Fonte: Elaborado pelas autoras.

Essas estratégias de monitoramento trouxeram segurança para a população e para a Estratégia Saúde da Família, uma vez que todos os pacientes com casos leves e sintomáticos respiratórios do território estavam 
sendo monitorados em tempo oportuno e de forma adequada, para minimizar os possiveis danos do não acompanhamento.

\section{Plantão Epidemiológico 24 horas}

As dificuldades vivenciadas durante a pandemia da Covid-19 motivaram o município de Sobral a inovar e buscar soluções eficazes para potencializar o compartilhamento de informações, orientações sobre isolamento e distanciamento social, bem como monitoramento dos casos. Dessa forma, o uso de tecnologias de informação e comunicação permitiu que as modalidades de atendimento on-line se tornassem as mais difundidas para a continuidade do cuidado e para responder às demandas dos usuários², além de representar uma estratégia de viabilizar a orientação à população sobre os cuidados necessários para a prevenção e tratamento da Covid-19.

Nesse contexto, após o Decreto Municipal $n .^{\circ}$ 2.371, de 16 de março de 2020, o município de Sobral entrou em estado de emergência em saúde decorrente da Covid-1910, iniciando, assim, uma série de ações com vistas a orientar a população, monitorar os casos suspeitos, minimizar os agravos e conter a disseminação do vírus no município. Dentre essas ações, inclui-se a implantação de um sistema de teleatendimento.

0 teleatendimento implementado em Sobral, também denominado Plantão Epidemiológico 24 horas, mantido pela Secretaria Municipal de Saúde, esteve ativo entre os meses de março a setembro de 2020. Tendo em vista a dinamicidade das necessidades da população nesse período, optouse, inicialmente, pela composição de uma equipe de atendimento multiprofissional composta por 16 profissionais, entre enfermeiros da APS, docentes da Escola de Saúde Pública Visconde de Saboia (ESP-VS) e profissionais do Nasf-AB. 0 Plantão Epidemiológico funcionava 24 horas, durante os sete dias da semana, com escala entre os profissionais.

Viabilizado por meio de uma multiplataforma de mensagens instantâneas, chamadas de voz ou vídeo e ligações, o Plantão Epidemiológico permitia o atendimento instantâneo a múltiplas pessoas. Ao final de cada plantão, todos os atendimentos eram registrados e arquivados por meio de ata e armazenamento em nuvem.

Entre as condutas indicadas via WhatsApp e telefone, destacam-se: orientações e direcionamentos ao Centro Municipal de Triagem da

\section{“O Plantão \\ Epidemiológico \\ permitia o atendimento instantâneo a múltiplas pessoas"}

Covid-19, aos Centros de Saúde da Família, à Unidade de Pronto Atendimento (UPA), medidas de isolamento domiciliar e outras orientações em relação a vacinas, consultas, exames, receitas, entre outras coisas que foram relevantes para que a população pudesse continuar a buscar cuidados prioritários à saúde de forma segura.

Serviços de atendimento on-line podem ser ferramentas importantes nos casos de pessoas com sintomas sugestivos de Covid-19 brandos ou com gravidade moderada ${ }^{11}$. Nesse contexto, esse serviço é uma estratégia de cuidado fundamental, possibilitando que os usuários tenham informação qualificada e em tempo oportuno de como proceder em nível individual.

No Brasil, as ações da Telessaúde já beneficiaram mais de um milhão de pessoas com orientações sobre sinais e sintomas do novo coronavírus. Desse total, 471,6 mil foram avaliadas a distância pelo Ministério da Saúde e aproximadamente 13 mil pessoas foram encaminhadas para teleatendimento pré-clínico com médicos, enfermeiros e técnicos de enfermagem ${ }^{12}$.

Além disso, o Plantão Epidemiológico 24 horas também acolhia denúncias relacionadas ao descumprimento do isolamento domiciliar de casos suspeitos ou confirmados. Essas denúncias eram comunicadas à Vigilância Sanitária e aos gerentes dos CSF para que fossem realizadas medidas necessárias a fim de interromper a cadeia de transmissibilidade do vírus.

Cabe destacar a importância deste trabalho em rede e da prática colaborativa entre os diversos segmentos das políticas públicas municipais, que foram fundamentais para o enfrentamento da pandemia em Sobral e para o cuidado e proteção da população em geral, principalmente daqueles que fazem parte do grupo de risco para a Covid-19.

No período de março a agosto de 2020, o Plantão Epidemiológico 24 horas realizou um total de 741 notificações de pessoas residentes no município de 
Sobral, como produto dos teleatendimentos realizados. Verificou-se que, dessas notificações, o pico de incidência de procura ocorreu nos meses de maio $(46,3 \% ; N=343)$ e junho $(28,00 \% ; N=207)$, que, consequentemente, corresponderam ao período com maior número de casos notificados de sintomáticos respiratórios. Esse dado nos faz pensar que esses dois meses corresponderam ao pico do número de casos de Covid-19 em Sobral. Essa informação também pode ser observada nos dados dos boletins epidemiológicos divulgados no site da Prefeitura Municipal de Sobral. Além disso, também registramos a redução da procura do serviço do teleatendimento a partir do mês de junho, acompanhado pela redução brusca dos registros de notificações de casos (Gráfico 1).

Gráfico 1 - Distribuição do número de casos notificados pelo Plantão Epidemiológico, de acordo com o mês de ocorrência. Sobral, Ceará, 2020.

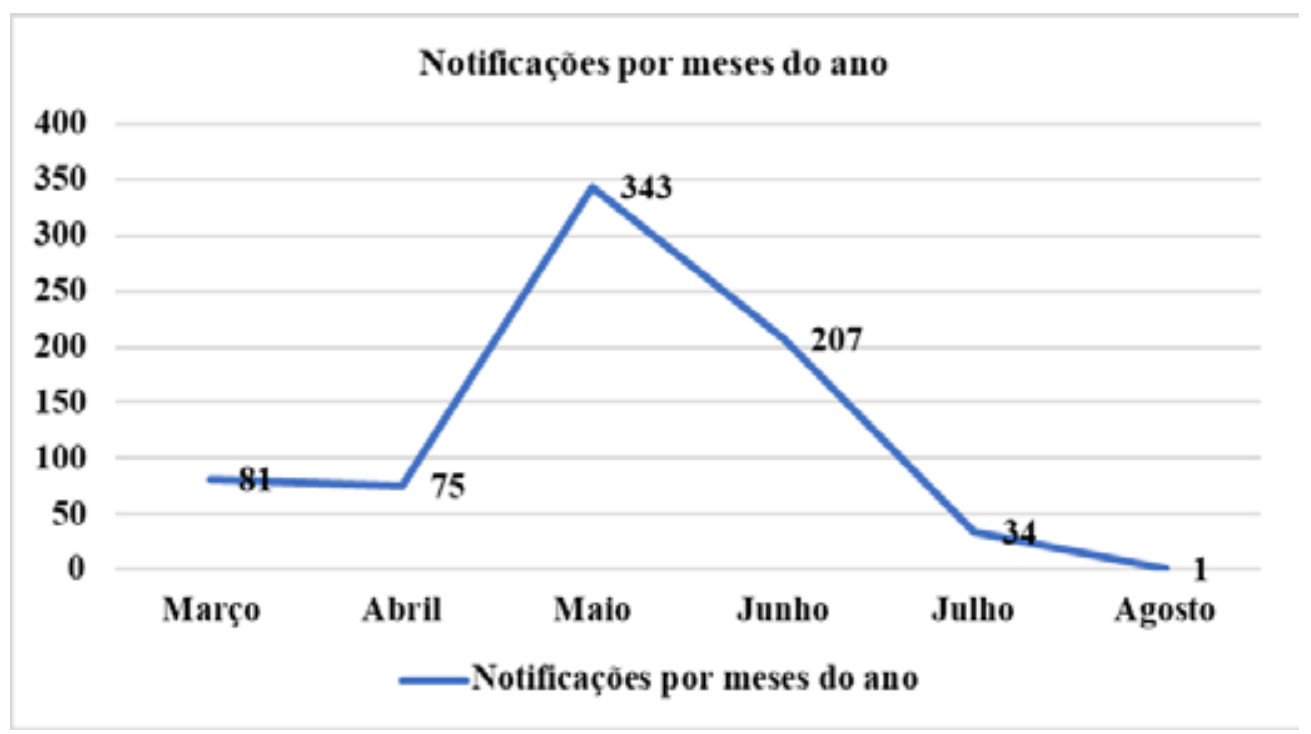

Fonte: Elaborado pelas autoras.

Das 741 notificações realizadas, $60,9 \%$ eram do sexo feminino $(n=451)$ e $39,1 \%(n=290)$ pertenciam ao sexo masculino. Sendo semelhante ao dado encontrado no estudo realizado no Triângulo Mineiro, em que $63,1 \%$ dos indivíduos eram do sexo feminino e $36,9 \%$ pertenciam a sexo masculino ${ }^{13}$. No que concerne à faixa etária, a maior procura por atendimento foi registrada no intervalo de 20 a 39 anos de idade, equivalente a um percentual de $44,1 \%(n=327)$. Esses dados são semelhantes aos encontrados no estado do Ceará, em que até 19 de março de $2020,42,7 \%$ dos suspeitos eram do sexo feminino, com idades entre 20 e 49 anos ${ }^{14}$.

Tendo em vista que a população adulta jovem é economicamente ativa, em sua maioria, muitas vezes essas pessoas precisavam continuar trabalhando para prover recursos a fim de sanar as demandas familiares essenciais; com isso, infere-se que esse fato contribuiu para a maior exposição ao vírus e, consequentemente, a um maior adoecimento delas.

Também foi observado um percentual de 19,2\% $(n=142)$ de notificações na faixa etária de 60 a 79 anos. Esse percentual é fruto de ações como orientações de isolamento e forte estímulo e cuidado às pessoas idosas. Entretanto, considerando as medidas adotadas para esse grupo, esperava-se um número menor.

Em relação aos sintomas notificados, os mais predominantes foram febre $(39,9 \% ; n=524)$, tosse $(30,8 \% ; n=$ 405) e dor de garganta $(19,9 \% ; n=252)$ (Gráfico 2), descritos como sendo os sinais e sintomas inespecíficos mais comuns nos casos confirmados de Covid-1912,15-16. Por serem facilmente confundidos com sintomas de resfriado comum, geram diversas dúvidas no que concerne aos sintomas da Covid-19 e, muitas vezes, causando pânico na população em geral. 
Gráfico 2 - Distribuição do número de casos notificados pelo Plantão Epidemiológico, de acordo com o mês de ocorrência. Sobral, Ceará, 2020.

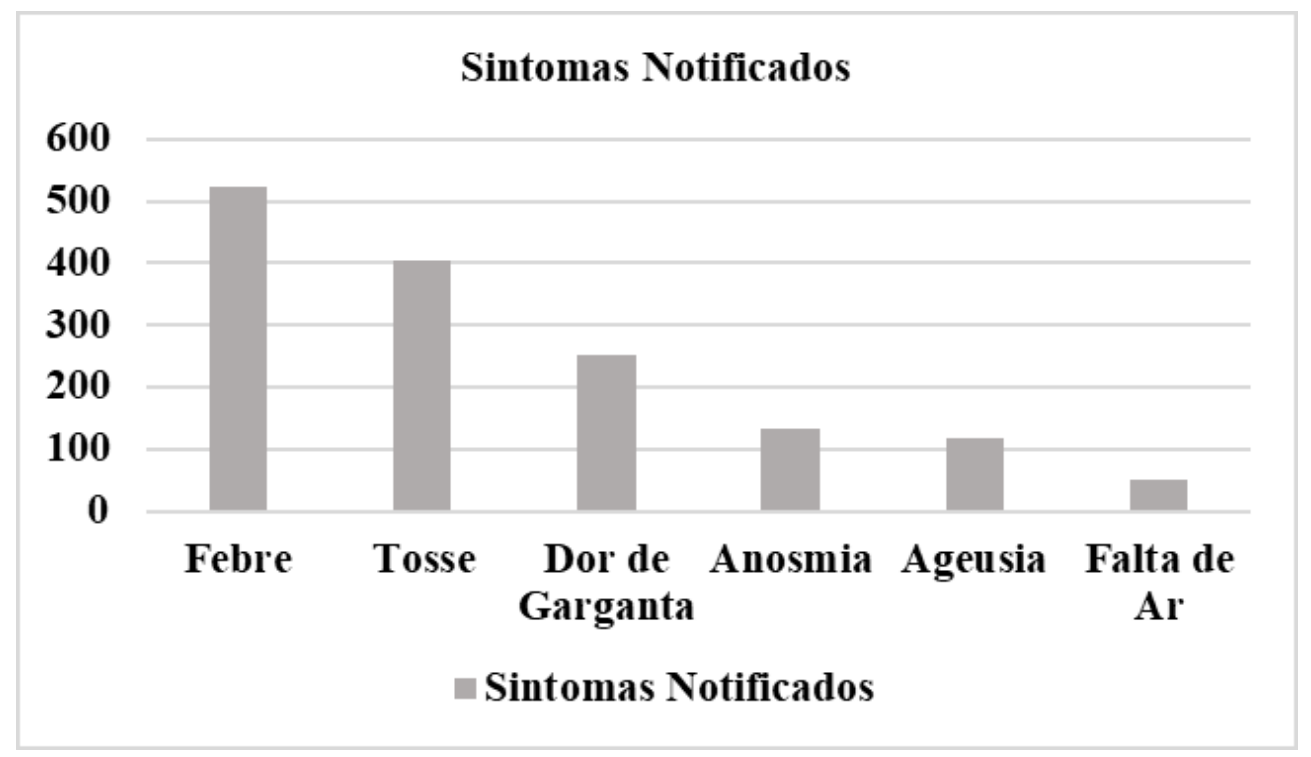

Fonte: Elaborado pelas autoras.

É importante destacar que pouco mais da metade $(51,8 \% ; \mathrm{N}=384)$ das notificações realizadas foram de pessoas do grupo de risco e que possuíam alguma comorbidade. Isso demonstra a grande preocupação dessas pessoas em buscar por um serviço, sem necessariamente deslocar-se, fisicamente, até ele; dessa forma, expondo-se ao minimamente necessário. Nesse ínterim, das comorbidades mais relatadas, 31,3\% dos indivíduos eram hipertensos $(n=232), 10,5 \%$ diabéticos $(n=78)$ e $9,9 \%$ asmáticos $(n=74)$. A partir disso, percebe-se a elevada notificação de pessoas com Doenças Crônicas não Transmissíveis (DCNT), em que as mais prevalentes são as cardiopatias, hipertensão, diabetes e doenças pulmonares crônicas. Tais grupos de risco se relacionam com índices de agravamento presentes, em sua maioria, nessa população ${ }^{17-18}$.

\section{CONCLUSÃO}

As experiências apresentadas foram fundamentais para subsidiar a tomada de decisão pela gestão municipal. Com isso, as estratégias utilizadas permitiram que toda a rede de saúde do município trabalhasse de forma articulada, dispondo de ferramentas de apoio norteadoras aos processos de trabalho durante a pandemia, por meio da prática colaborativa entre os serviços de saúde e demais serviços do âmbito municipal.

As variadas formas de monitoramento apresentam relevância no que condiz à orientação dos profissionais de saúde para a adoção de novos protocolos sanitários e para a identificação, manejo, prevenção e tratamento de sintomas respiratórios oriundos da Covid-19.

É significativo destacar a importância das estratégias de monitoramento para o acompanhamento integral das condições de saúde da população, para a identificação e tratamento precoces dos sintomas respiratórios, para a Vigilância Epidemiológica e notificação dos casos suspeitos aos órgãos estaduais e federais da saúde, para a ampliação do acesso à informação em meio a medidas de isolamento rígido, para o rastreio dos contatos e prevenção da transmissibilidade da doença, bem como para a garantia de atendimento via ferramentas digitais com vistas ao enfrentamento da Covid-19 no município de Sobral.

Essas ações exigiram alto investimento da gestão municipal em recursos humanos e tecnológicos. Com isso, à medida que crescia a curva de contágio, faziam-se necessários um maior número de profissionais trabalhando e uma maior quantidade de equipamentos de informática.

Ressalta-se como grande desafio para a execução das estratégias de monitoramento a necessidade de capacitações constantes para os profissionais de saúde no que se refere ao uso das ferramentas e 
plataformas digitais, à compreensão dos protocolos sanitários, ao manejo clínico dos casos suspeitos e às recomendações das autoridades sanitárias estaduais para a contenção da doença.

A experiência do monitoramento em Sobral teve como limitação a dificuldade na comunicação de dados entre a rede hospitalar e a APS, que foi realizada por meio de contato telefônico ou planilhas editáveis pelos profissionais da Central de Monitoramento, sendo essas informações repassadas, muitas vezes, tardiamente.

Essa dificuldade poderia ter sido superada com a criação de um software que permitisse que os profissionais responsáveis pelo monitoramento da APS pudessem ter acesso on-line aos dados de acompanhamento, evolução, óbito e alta dos pacientes com Covid-19 que eram atendidos em serviços de alta complexidade, no intuito de acompanhar em tempo oportuno os familiares e prevenir complicações ou sequelas por Covid-19 em pacientes com alta hospitalar.

Por intermédio das estratégias apresentadas, evidenciou-se a importância da reorganização da rede de saúde, utilizando as tecnologias da informação para investir em monitoramento de casos, rastreamento de contatos, orientações preventivas para a população em geral e capacitação dos profissionais de saúde. Isso colabora para a vigilância dos dados epidemiológicos e subsidia a tomada de decisão dos gestores municipais. Também ajuda a direcionar melhor as ações de prevenção nos territórios onde há um crescimento de novas infecções. Percebeu-se que os esforços preventivos são mais eficazes quando associados às medidas de monitoramento e vigilância.

\section{CONTRIBUIÇÃO DAS AUTORAS}

Josiane Alves Dorneles contribuiu com redação, análise e revisão crítica do manuscrito. Rogeriany Lopes Farias contribuiu com redação, análise e revisão crítica do manuscrito. Larissa Cavalcante Fonteles Araújo contribuiu com redação, análise e revisão crítica do manuscrito. Paloma de Vasconcelos Rodrigues contribuiu com redação, análise e revisão crítica do manuscrito. Isabelle Frota Ribeiro Queiroz contribuiu com redação, análise e revisão crítica do manuscrito. Germana Maria da Silveira contribuiu com redação, análise e revisão crítica do manuscrito.

\section{REFERÊNCIAS}

1. Medina MG, Giovanella L, Bousquat A, Mendonça MHM, Aquino R. Atenção primária à saúde em tempos de COVID-19: o que fazer? Cad. Saúde Publica [serial on the internet]. 2020 [cited 2020 Nov 13];36(8): e00149720. Available from: https:// www.scielo.br/pdf/csp/v36n8/1678-4464-csp-3608-e00149720.pdf

2. Aquino $E$, Silveira IH, Pescarini J, Aquino R, Souza-Filho JA. Medidas de distanciamento social no controle da pandemia de COVID-19: potenciais impactos e desafios no Brasil. Cien Saúde Colet [serial on the internet]. 2020 [cited 2020 Nov 13];25(Suppl 1):2423-46. Available from: https://www.scielo.br/scielo.php?pid=S1413$81232020006702423 \&$ script $=$ sci arttext

3. Brasil. Guia de Vigilância Epidemiológica/ Emergência de Saúde Pública de Importância Nacional pela Doença pelo Coronavírus 2019/ Vigilância de Síndromes Respiratórias Agudas COVID-19 [document on the internet]. Brasilia (DF): Ministério da Saúde; 2020 [cited 2020 Nov 29]. Available from: http://www.dive.sc.gov.br/ notastecnicas/docs/af gvs coronavirus 6 ago 2 0 ajustes-finais-2.pdf

4. Silva DAJ, Tavares SMFL. Ação intersetorial: potencialidades e dificuldades do trabalho em equipe da Estratégia de Saúde da Família na cidade do Rio de Janeiro. Rev Saúde Debate [serial on the internet]. 2016 [cited 2020 Nov 27];40(111):193205. Available from: https://www.scielo. $\mathrm{br} / \mathrm{pdf} / \mathrm{sdeb} / \mathrm{v} 40 \mathrm{n} 111 / 0103-1104-\mathrm{sdeb}-40-111-0193$. pdf

5. Organização Pan-Americana da Saúde. OPAS pede aos países que reforcem rastreamento de contatos e sistemas de dados à medida que a região começa a reabrir [document on the internet]. Brasília (DF): OPAS [cited 2020 Nov 29]. Available from: https://www.paho.org/bra/index.php?opt ion $=$ com content \&view $=$ article \&id $=6261:$ opaspede-aos-paises-que-reforcem-rastreamento-decontatos-e-sistemas-de-dados-a-medida-que-aregiao-comeca-a-reabrir\&Itemid $=812$

6. Barbosa SP, Silva AVFG. A Prática da Atenção Primária à Saúde no Combate da Covid-19. APS em Revista [serial on the internet]. 2020 [cited 2020 Nov 29];2(1):17-9. Available from: https://apsemrevista. org/aps/article/view/62/43

7. Starfield B. Atenção primária: equilíbrio entre a necessidade de saúde, serviços e tecnologias. Brasília (DF): UNESCO, Ministério da Saúde; 2002.

8. Brasil. Coronavírus Covid-19: Fast-track para a atenção primária em locais com transmissão comunitária fluxo rápido: versão 6. Brasília (DF): Ministério da Saúde; 2020. 
9. Brasil. Recomendações para adequação das ações dos Agentes Comunitários de Saúde frente à atual situação epidemiológica referente ao Covid-19: versão 1. Brasília (DF): Ministério da Saúde; 2020.

10. Sobral. Decreto Municipal $n^{\circ} 2.371$, de 16 de março de 2020. Decreta estado de emergência no âmbito do município de sobral e estabelece medidas para enfrentamento do novo coronavírus (COVID-19), e dá outras providências [serial on the internet]. Diário Oficial do Município, Brasília (2020 Mar 16 [cited 2020 Nov 29]); Sec 1.

11. Greenhalgh T, Wherton J, Shaw S, Morrison C. Video consultations for covid-19. BMJ [serial on the internet]. 2020 [cited 2020 Nov 29];368:m998. Available from: https://www.bmj.com/con tent/368/bmj.m998 _

12. Brasil. Programa Nacional Telessaúde Brasil Redes [document on the internet]. Brasília (DF): Ministério da Saúde; 2020 [cited 2020 Nov 29]. Available from: https://aps.bvs.br/programa-nacional-telessaudebrasil-redes/

13. Rezende AP, Camargo RC, Santana VF, Escoura LD, Melo MA, Rosin LP, et al. A contribuição do teleatendimento para o combate da COVID-19. SanarMed [serial on the internet]. 2020 [cited 2020 Nov 29];3:6-10. Available from: https:// www.researchgate.net/profile/Andre Fukus $\mathrm{hima/publication/344524024}$ RevistaSanarMedvol3sanarcon/ links/5f7e205392851c14bcb66f64/RevistaSanarMed-vol3-sanarcon.pdf\#page $=7$

14. Ceará. Boletim epidemiológico: Doença pelo novo coronavírus (COVID-19) $\mathrm{n}^{\circ} 18$ [document on the internet]. Ceará: Secretaria da Saúde do Estado do Ceará; 2020 [cited 2020 Nov 29]. Available from: https://www.saude.ce.gov.br/ wpcontent/uploads/sites/9/2018/06/boletim n18 COVID 19 mar 20 SM.pdf

15. Li C, Ji F, Wang L, Wang L, Hao J, Dai M, et al. Asymptomatic and human-to-human transmission of SARS-CoV-2 in a 2-family cluster, Xuzhou, China. Emerg. Infect Dis [serial on the internet]. 2020 [cited 2020 Nov 29];26(7):1626-28. Available from: https://www.ncbi.nlm.nih.gov/ pmc/articles/PMC7323514/

16. World Health Organization. Coronavirus disease (COVID-19) pandemic [internet]. Geneva: WHO； 2020 [cited 2020 Nov 29]. Available from: https://www.who.int/emergencies/dise ases/novel-coronavirus-2019

17. Brasil. Diretrizes para diagnóstico e tratamento da Covid-19 [document on the internet]. Brasília (DF): Ministério da Saúde; 2020 [cited 2020 Nov 13]. Available from: https://portaldeboaspraticas. iff.fiocruz.br/atencao-mulher/diretrizes-paradiagnostico-e-tratamento-da-covid-19-ms/
18. Brasil. Protocolo de manejo clínico do coronavírus (COVID-19) na Atenção Primária à Saúde [document on the internet]. Brasília (DF): Ministério da Saúde; 2020 [cited 2020 Nov 13]. Available from: http://www.nutes.ufpe.br/indu/mod/resource/view. php?id=14691
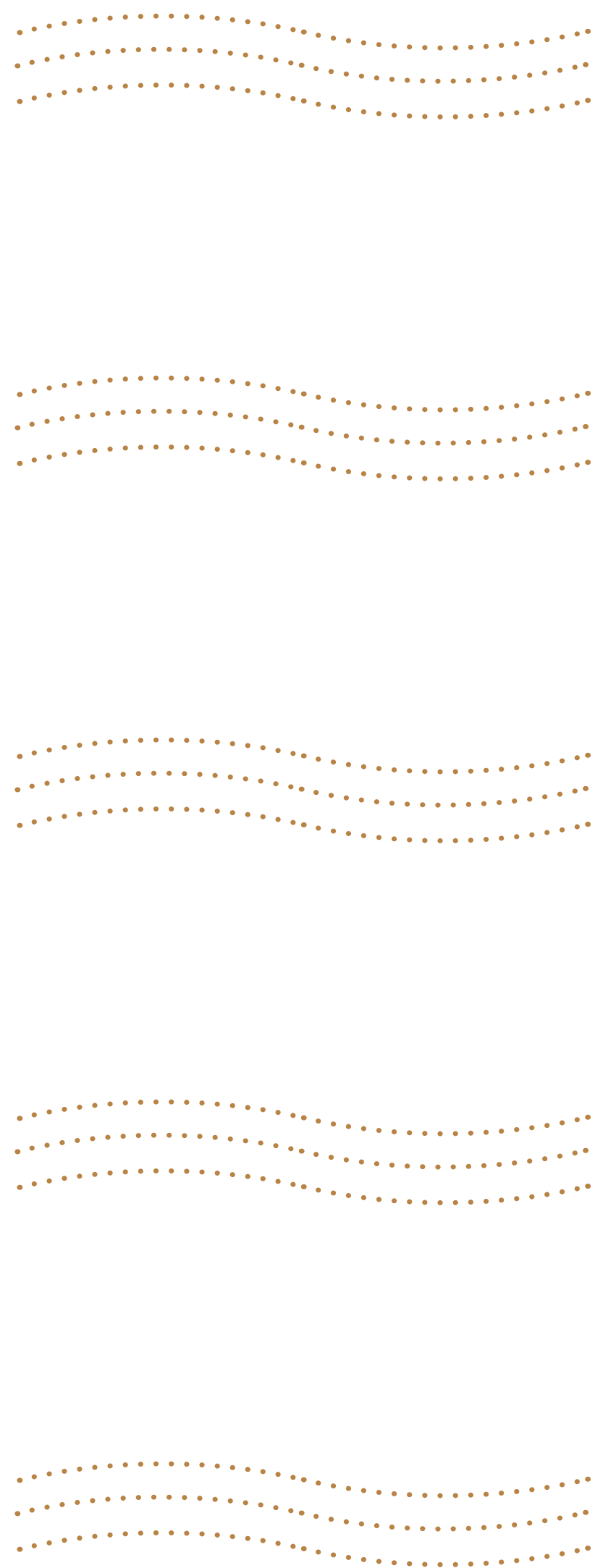

SANARE (Sobral, online). 2021; 20 (Supl. 1): 71-81 - 81 